DiRECTEUR DE LA PUBLICATION / PUBLICATION DIRECTOR: Bruno David,

Président du Muséum national d'Histoire naturelle

RÉDACTEUR EN CHEF / EDITOR-IN-CHIEF: Jean-Philippe Siblet

ASSISTANTE DE RÉDACTION / ASSISTANT EDITOR: Sarah Figuet (naturae@mnhn.fr)

Mise EN PAGE / PAGE LAYOUT: Sarah Figuet

COMITÉ SCIENTIFIQUE / SCIENTIFIC BOARD:

Luc Abbadie (UPMC, Paris)

Luc Barbier (Parc naturel régional des caps et marais d'Opale, Colembert)

Aurélien Besnard (CEFE, Montpellier)

Vincent Boullet (Expert indépendant flore/végétation, Frugières-le-Pin)

Hervé Brustel (École d'ingénieurs de Purpan, Toulouse)

Patrick De Wever (MNHN, Paris)

Thierry Dutoit (UMR CNRS IMBE, Avignon)

Éric Feunteun (MNHN, Dinard)

Romain Garrouste (MNHN, Paris)

Grégoire Gautier (DRAAF Occitanie, Toulouse)

Olivier Gilg (Réserves naturelles de France, Dijon)

Frédéric Gosselin (Irstea, Nogent-sur-Vernisson)

Patrick Haffner (UMS PatriNat, Paris)

Frédéric Hendoux (MNHN, Paris)

Xavier Houard (OPIE, Guyancourt)

Isabelle Le Viol (MNHN, Concarneau)

Francis Meunier (Conservatoire d'espaces naturels - Hauts-de-France, Amiens)

Serge Muller (MNHN, Paris)

Francis Olivereau (DREAL Centre, Orléans)

Laurent Poncet (UMS PatriNat, Paris)

Nicolas Poulet (OFB, Vincennes)

Jean-Philippe Siblet (UMS PatriNat, Paris)

Laurent Tillon (ONF, Paris)

Julien Touroult (UMS PatriNat, Paris)

COUVERTURE / COVER:

Corbicule asiatique Corbicula fluminea (O. F. Müller, 1774). Crédit photo: C. Lemarchand.

\section{MusćuM * Nantes

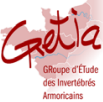

Les Publications scientifiques du Muséum publient aussi / The Museum Science Press also publish:

Adansonia, Zoosystema, Anthropozoologica, European Journal of Taxonomy, Geodiversitas, Cryptogamie sous-sections Algologie, Bryologie, Mycologie, Comptes Rendus Palevol.

Diffusion - Publications scientifiques Muséum national d'Histoire naturelle

CP 41 - 57 rue Cuvier F-75231 Paris cedex 05 (France)

Tél. : 33 (0)1 40794805 / Fax: 33 (0)1 40793840

diff.pub@mnhn.fr / http://sciencepress.mnhn.fr

(C) Publications scientifiques du Muséum national d'Histoire naturelle, Paris, 2020

ISSN (électronique / electronic): 1638-9387 


\title{
Identification de Corbicules (Corbicula sp. Müller, 1774) dans les collections étudiées par le Muséum Henri-Lecoq
}

\author{
Charles LEMARCHAND \\ Muséum Henri-Lecoq, 15 rue Bardoux, F-63000 Clermont-Ferrand (France) \\ clemarchand@clermontmetropole.eu
}

Soumis le 10 juillet 2019 | Accepté le 8 novembre 2019 | Publié le 9 novembre 2020

MOTS CLÉS

Corbicula fuminea Corbicula fluminalis, collections muséales.

Lemarchand C. 2020. - Identification de Corbicules (Corbicula sp. Müller, 1774) dans les collections étudiées par le Muséum Henri-Lecoq, in Léonard L. (éd.), Colloque national de malacologie continentale, Nantes, 6 et 7 décembre 2018. Naturae 2020 (12): 185-188. https://doi.org/10.5852/naturae2020a12

\section{RÉSUMÉ}

Les Corbicules, et notamment la Corbicule asiatique Corbicula fluminea (O. F. Müller, 1774) ont, ces dernières années, très rapidement colonisé plusieurs grands bassins hydrographiques en France (Loire, Garonne, Rhône) et en Europe, comme dans d'autres foyers d'introduction. En 2018, l'étude de collections malacologiques de l'université Clermont Auvergne par l'équipe du Muséum HenriLecoq de Clermont Auvergne Métropole a permis d'identifier des Corbicules parmi des spécimens de mollusques issus de divers bassins d'Europe de l'Ouest datant de la fin du XIXe siècle, alors que l'on considérait leur introduction comme beaucoup plus récente. L'intérêt de l'étude des collections naturalistes dans la compréhension de la dynamique des espèces introduites s'en trouve ainsi confirmé.

\section{ABSTRACT}

Asian clams (Corbicula sp. Müller, 1774) identification in collections studied by the Museum Henri-Lecoq (Clermont Auvergne Métropole, France).

In recent years, Asian clams and especially Corbicula species (C. Auminea (O. F. Müller, 1774) and $C$. fluminalis (O. F. Müller, 1774)) have widely and rapidly colonized several large river basins in France (Loire, Garonne, Rhône) and in Europe, as in other loci of introduction. In 2018, malacological study of collections of Clermont Auvergne University by the team of the Museum Henri-Lecoq (Clermont Auvergne Métropole) allowed to identify Asian clams among specimens of shellfish coming from

KEY WORDS

Corbicula fuminea Corbicula fluminalis, Museum collections. various basins of Western Europe. They were dated from the late nineteenth century, when Asian clams introduction was considered much more recent. The interest of studying naturalist collections in understanding the dynamics of introduced species is thus confirmed. 


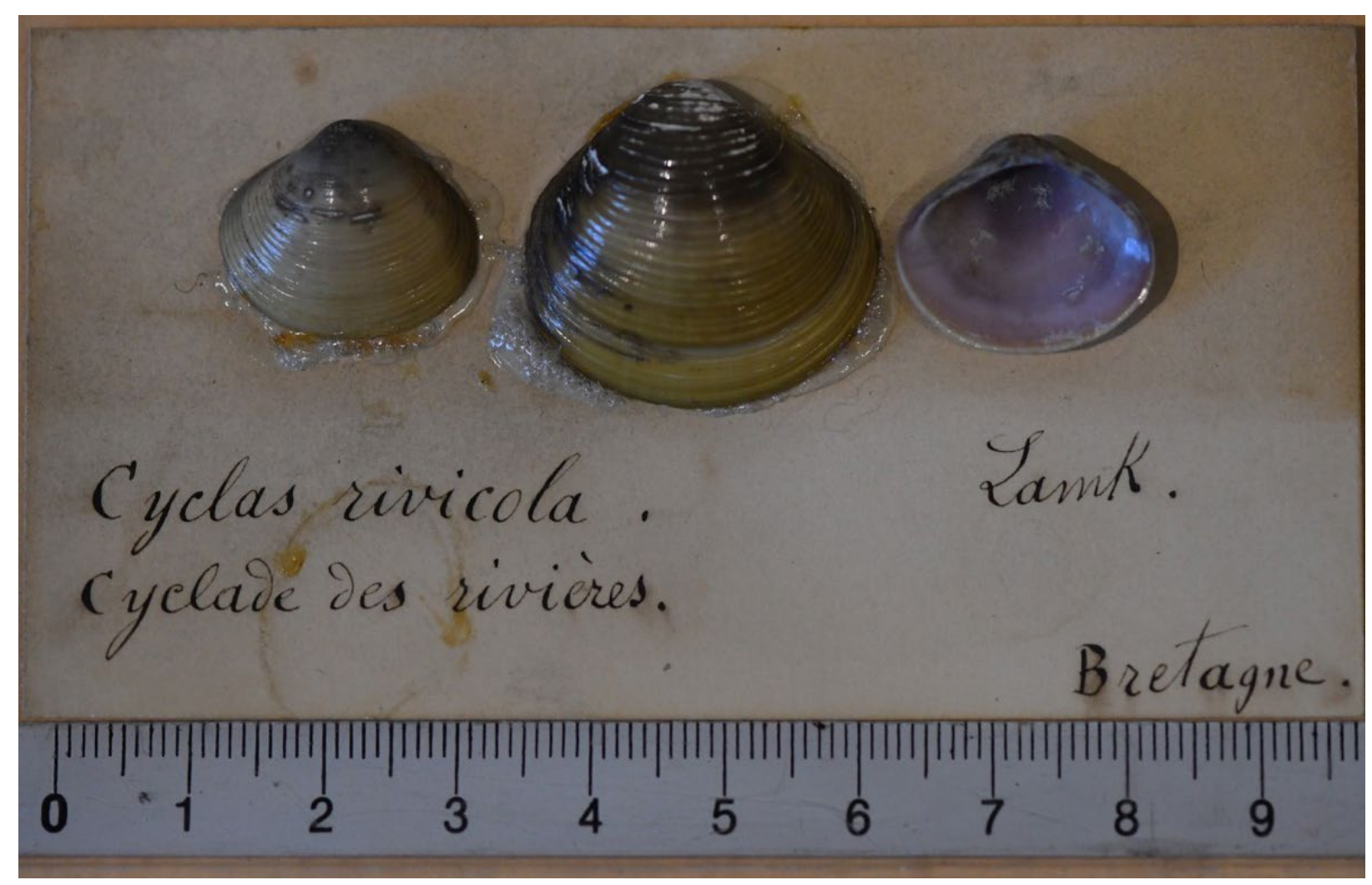

FIG. 1. - Corbicules issues de la collection de M. Vergne et identifiées comme des Cyclades des rivières. Crédit photo: C. Lemarchand, Muséum Henri-Lecoq, Clermont Auvergne Métropole.

\section{LES CORBICULES EN FRANCE}

Les Corbicules (Cyrenidae Gray, 1840) actuellement présentes en Europe, originaires d'Asie du Sud-Est, ont vraisemblablement été introduites involontairement par les activités humaines, notamment via les eaux de ballast des bateaux provenant d'Asie ou d'Amérique du Nord (où elles sont mentionnées dès 1924; Vidal 2001). En Europe, plusieurs espèces ont été recensées, dont la Corbicule striolée Corbicula fluminalis (O. F. Müller, 1774), la Corbicule asiatique Corbicula fluminea (O. F. Müller, 1774) et la Corbicule japonaise Corbicula leana (Prime, 1867). Ces deux dernières sont les plus répandues en France et, étant difficiles à identifier avec précision sur le terrain, elles sont généralement regroupées sous le vocable et la détermination unique de Corbicule asiatique Corbicula fluminea (Prié 2017). Absentes des gisements fossiles de l'Holocène en Europe de l'ouest, les Corbicules (notamment une variété polymorphe de C. fluminalis) ont toutefois été documentées dans des gisements correspondant aux périodes tempérées du Pléistocène (Meijer \& Preece 2000). Ces découvertes suggèrent de grandes variations de la distribution des Corbicules au sein du Paléarctique au fil des temps géologiques.

Les premières mentions au sein du réseau hydrographique français datent de la fin des années 1970 dans le Val de Loire. Au cours des années 1980, les Corbicules sont signalées dans la basse vallée de la Dordogne (Brancotte \& Vincent 2002; Prié 2017). Leur expansion est ensuite très rapide puisque dès 1985 elles sont signalées dans la Garonne (Brancotte \& Vincent 2002), puis dans le canal du Midi en 1989 (Girardi 1990). En 1990, leur présence est rapportée dans l'estuaire de la Loire (Brancotte \& Vincent 2002), dans les années 2000 sur la Loire moyenne (Brancotte \& Vincent 2002) et plus récemment sur la rivière Allier (Vrignaud 2007), soit plus de $700 \mathrm{~km}$ parcourus en une vingtaine d'années seulement au sein du bassin de la Loire. Leur association avec les activités humaines, particulièrement les flux de transport par bateaux, les rend très adaptées à la colonisation de nouveaux milieux (Brancotte \& Vincent 2002; Devin et al. 2005; Sousa et al. 2008), même si elles sont parfois moins résistantes aux pollutions que certaines espèces natives (Faria et al. 2010) et moins longévives.

L'observation de la présence d'une espèce non autochtone à l'état sauvage sur le terrain peut parfois se produire longtemps après son introduction, tout particulièrement dans le cas d'espèces difficiles ou délicates à déterminer par des non-spécialistes ou vivant dans des environnements peu échantillonnés par les naturalistes et les scientifiques. L'utilisation des connaissances liées à des inventaires de sites ou à la constitution de collections, réalisées par des Muséums, des associations, des services de l'État, des bureaux d'études ou encore des particuliers, peut alors permettre de déterminer une antériorité de présence, voire d'affiner une époque d'introduction ou une répartition diachronique. Concernant les Corbicules par exemple, Prié (2017) cite des individus présents dans une collection de la DREAL Centre, provenant du Val de Loire et datée de la fin des années 1970. 


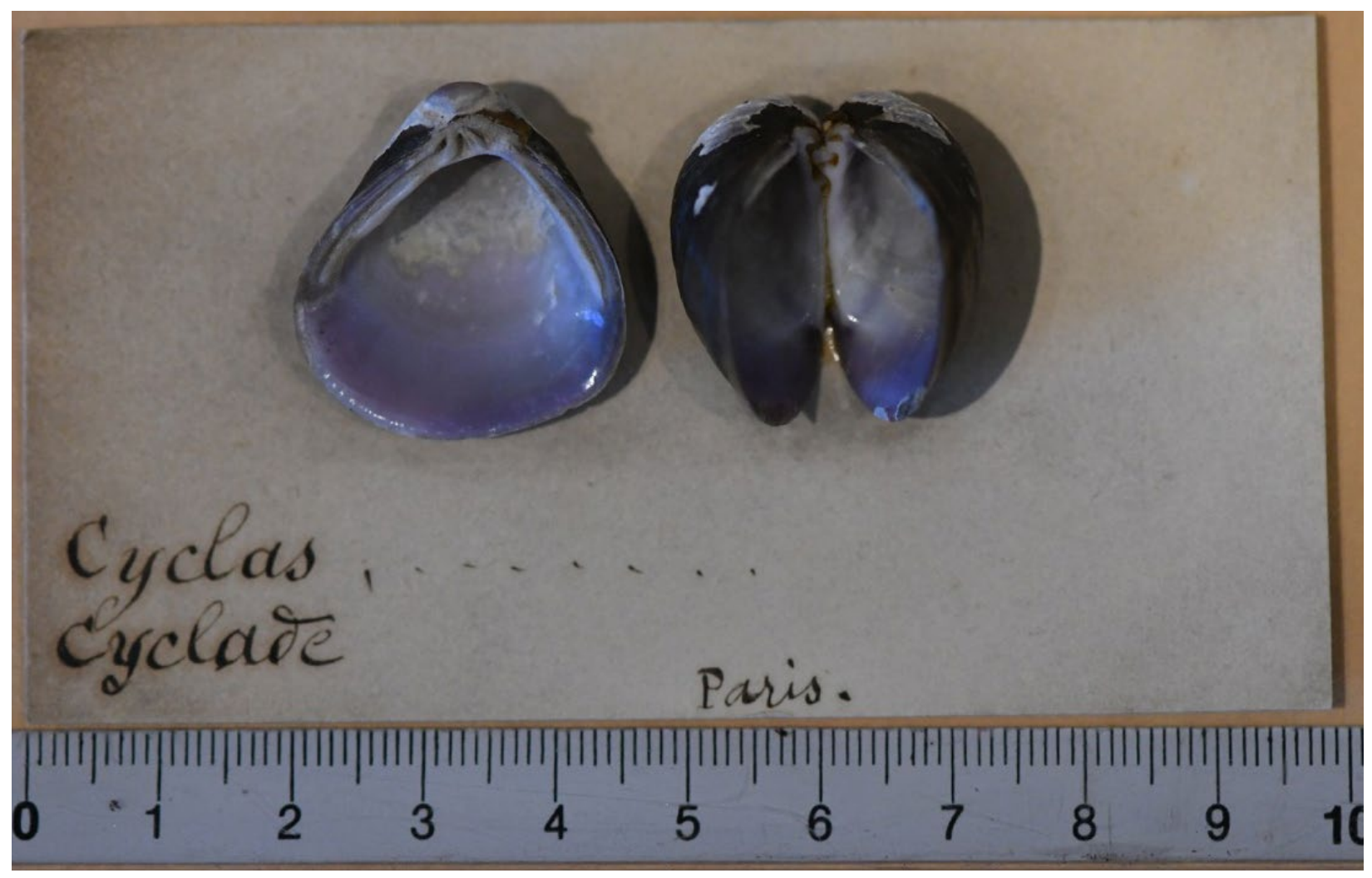

FIG. 2. - Corbicules issues de la collection de M. Vergne et identifiées comme des Cyclades. Crédit photo: C. Lemarchand/Muséum Henri-Lecoq/Clermont Auvergne Métropole.

\section{DES CORBICULES DANS UNE COLLECTION UNIVERSITAIRE DU MUSÉUM HENRI-LECOQ}

L'origine des collections zoologiques de l'université BlaisePascal de Clermont-Ferrand remonte au XIXe siècle, à la fondation du Muséum d'Histoire naturelle de la ville par l'abbé Lacoste. Henri Lecoq en a été le conservateur municipal jusqu'en 1860, date à laquelle la ville dépose officiellement ses collections à l'université nouvellement créée (1854). Lecoq est resté responsable des collections mais au titre de titulaire de la chaire d'histoire naturelle de l'université. L'enrichissement des collections s'est depuis poursuivi pour servir principalement à l'illustration des enseignements.

De nos jours, l'université Clermont Auvergne et Clermont Auvergne Métropole assurent de façon concertée le dépôt, l'étude, l'inventaire, la conservation, la restauration et l'exposition des collections universitaires (Charles \& Faure 2016). Ces collections, déposées en plusieurs lots, rassemblent plusieurs milliers de spécimens appartenant à différents rangs taxonomiques. On y distingue par exemple la collection de Michel Vergne (1893-1985), originaire de la région Auvergne. Diplômé en pharmacie et titulaire d'un doctorat ès sciences à la Sorbonne, il a enseigné à la faculté de Médecine et de Pharmacie de Clermont-Ferrand et a parallèlement été, de 1932 à sa retraite, conservateur des collections zoologiques du Musée Henri-Lecoq. En plus de sa vie professionnelle, Michel Vergne a consacré beaucoup de temps à la constitution de collections, essentiellement entomologiques mais aussi malacologiques. Une partie des premières ont été données au Muséum Henri-Lecoq par la famille de M. Vergne en 1997, une seconde partie de ses collections, dont les spécimens de malacologie ayant fait l'objet d'un dépôt universitaire en 2016. Cette collection, n'ayant pas fait l'objet d'entretien ni d'inventaire récent, se trouvait dans un état d'empoussièrement assez avancé au moment de son dépôt, et aucun document descriptif (catalogue, inventaire, etc.) n’y était associé.

Depuis le dépôt universitaire, les étudiants de l'université Clermont Auvergne peuvent travailler sur les collections de leur propre établissement, avec les techniques muséales au sein du Muséum. C'est au cours d'une des séances de travail sur les collections malacologiques de M. Vergne, consistant en un dépoussiérage manuel et une vérification taxonomique des spécimens, que l'attention d'un groupe d'étudiantes a été attirée. Plusieurs spécimens portaient en effet des mentions relatives à des "Cyclades" (Figs 1, 2), sous une forme indéterminée à l'espèce en général. Ces spécimens, issus de plusieurs localités géographiques différentes, n'étaient par ailleurs pas systématiquement renseignés quant à leur date de collecte.

Après une vérification approfondie à l'aide de clés de détermination et de loupes binoculaires, qui n'a toutefois pu concerner que les faces visibles en raison du risque de dégradation de l'ensemble en cas de décollement de l'étiquette portoir, les spécimens en question ont pu être déterminés et identifiés comme des Corbicules, appartenant potentiellement aux espèces $C$. fluminea et $C$. leana (Figs 1, 2), parfois en 
mélange avec des Cyclades (confirmant le risque de confusion entre ces taxons). La localisation géographique des spécimens est intéressante. Elle correspond en effet à deux ensembles différents, le bassin de la Seine (Paris) d'une part, la Bretagne d'autre part, sans qu'une localisation fine (portion de la Seine, rivière ou fleuve côtier en Bretagne) ne puisse être effectuée. Malheureusement, aucune des étiquettes portoirs des spécimens identifiés comme des Corbicules ne comportait de date de récolte ou de mise en collection, compromettant la richesse de l'information scientifique associée. Cependant, d'après l'écriture manuscrite lisible sur les étiquettes, leur type, leur degré d'empoussièrement et leur mise en collection aux côtés d'autres spécimens correctement datés et de présentation comparable, nous pouvons en déduire que ces spécimens datent très vraisemblablement de la période comprise entre le dernier quart du XIXe siècle et le premier quart du XXe. Malgré ce manque de précision, cette mention de Corbicules en collections apporte la preuve que l'introduction de ces espèces et leur intégration à la faune sauvage française, voire européenne, remonte potentiellement à la seconde moitié du XIXe siècle, soit près d'un siècle avant les périodes précédemment avancées.

\section{CONCLUSION}

L'étude de la collection de M. Vergne se poursuit, afin notamment de tenter de découvrir d'autres spécimens de Corbicules ou davantage d'informations sur celles déjà identifiées. Ce travail confirme également la nécessité de réaliser des enquêtes auprès des détenteurs de collections malacologiques afin de fixer plus finement les périodes et les localités d'introduction des Corbicules, mais aussi d'autres espèces de Mollusques (Prié 2017).

\section{Remerciements}

L'auteur remercie les rapporteurs pour leurs remarques constructives ayant permis d'améliorer la lisibilité du texte, ainsi que
Manon Chaussidon et Emma Viallet pour leur travail de tri, d'inventaire et de conservation des collections.

\section{RÉFÉRENCES BIBLIOGRAPHIQUES}

BRANCOTTE V. \& Vincent T. 2002. - L'invasion du réseau hydrographique français par les Mollusques Corbicula spp.: modalités de colonisation et rôle prépondérant des canaux de navigation. Bulletin français de la Pêche et de la Pisciculture 365/366: 325-337. https://doi.org/10.1051/kmae:2002038

Charles L. \& FAURE M.-F. 2016. — La collection malacologique d'Henri Lecoq et les Coquilles de P. L. Duclos. (Coll. Muséum Henri-Lecoq, VII). Muséum Henri-Lecoq, Clermont Auvergne Métropole, Clermont-Ferrand, 128 p.

Devin S., Bollache L., NoËL P.-Y. \& Beisel J.-N. 2005. Patterns of biological invasions in French freshwater systems by non-indigenous macroinvertebrates. Hydrobiologia 551 (1): 137-146. https://doi.org/10.1007/s10750-005-4456-z

Faria M., Angel Lopez M., Diez S. \& Barata C. 2010. — Are native naiads more tolerant to pollution than exotic freshwater bivalve species? An hypothesis tested using physiological responses of three species transplanted to mercury contaminated sites in the Ebro River (NE, Spain). Chemosphere 81 (10): 12181226. https://doi.org/10.1016/j.chemosphere.2010.09.037

GIRARDi H. 1990. - Deux bivalves d'eau douce récents pour la faune française (Mollusca, Bivalvia). Bulletin de la Société de Science Naturelles du Vaucluse 1: 87-93.

Meijer T. \& Preece R. C. 2000. - A review of the occurrence of Corbicula in the Pleistocene of North-West Europe. Geologie en Mijnbouw / Netherlands Journal of Geosciences 79 (2-3): 241-255. https://doi.org/10.1017/S0016774600021739

PRIÉ V. 2017. - Nä̈ades et autres bivalves d'eau douce de France. Biotope, Mèze, $336 \mathrm{p}$.

Sousa R., Antunes C. \& Guilhermo L. 2008. - Ecology of the invasive Asian clam Corbicula fluminea (Müller, 1774) in aquatic ecosystems: an overview. Annals of Limnology International Journal of Limnology 44 (2): 85-94. https://doi. org/10.1051/limn:2008017

VIDAL M. L. 2001. - Étude de marqueurs biochimiques de pollution chez le mollusque bivalve d'eau douce Corbicula fluminea (Müller, 1774) - Purification et caractérisation des glutathion$S$-transférases. Thèse de doctorat, Université Bordeaux I, Bordeaux, 284 p.

VRIGNAUD S. 2007. - Corbicules. Margaritifera - Bulletin de liaison de l'atlas des Mollusques de l'Allier 6: 1-15. 